\title{
Least Action Principles and Well-Posed Learning Problems
}

\author{
Alessandro Betti and Marco Gori
}

\begin{abstract}
Machine Learning algorithms are typically regarded as appropriate optimization schemes for minimizing risk functions that are constructed on the training set, which conveys statistical flavor to the corresponding learning problem. When the focus is shifted on perception, which is inherently interwound with time, recent alternative formulations of learning have been proposed that rely on the principle of Least Cognitive Action, which very much reminds us of the Least Action Principle in mechanics. In this paper, we discuss different forms of the cognitive action and show the well-posedness of learning. In particular, unlike the special case of the action in mechanics, where the stationarity is typically gained on saddle points, we prove the existence of the minimum of a special form of cognitive action, which yields forth-order differential equations of learning. We also briefly discuss the dissipative behavior of these equations that turns out to characterize the process of learning.
\end{abstract}

\section{Introduction}

Whenever a learning process is embedded in a temporal environment; i.e. the data presented to the agent has a temporal structure (video and audio signals for example) it seems natural to define the learning process directly through the definition of a suitable temporal dynamics. In other words one might start to think that the updating of the model's parameters, which is what we usually call "learning", must be synced with the temporal structure of data. This suggests investigating the continuous map $t \mapsto w(t)$ as a response to the input $u(t)$, thus regarding $t$ as time and not simply an iteration index of popular machine learning algorithms.

Alessandro Betti

DINFO, Via di S. Marta, 3 Firenze, Italia, e-mail: alessandro.betti@unifi.it

Marco Gori

DIISM, via Roma, 56, Siena, Italia e-mail:marco@dii.unisi.it 
In order to be able to select the correct dynamics of the weights of an agent we believe that a functional formulation of the problem is particularly useful. For example, the Lagrangian formulation of physical theories offers the possibility of imposing all the symmetries of a theory simply adding to the Lagrangian terms that satisfy such symmetry (see for example [1]). In the same way [2], this approach makes it easier to incorporate constraints on the dynamic of the learned weights. A variational approach based on an integral functional like the action of classical mechanics can be conceived which specifies in one single scalar function (what in mechanics is called the Lagrangian) both the "static" goodness criterion, the potential, and the dynamical part of learning by a kinetic term [3].

For example, consider a classical batch problem in machine learning where the functional risk has been approximated with a function $V(w)$. As we will discuss in Section 2 we can find appropriate functional indexes that have as stationarity condition the following differential equation

$$
m \ddot{w}+\eta \dot{w}+\nabla V(w)=0 \quad m, \eta>0 .
$$

This equation can be considered as the continuous form of a classic multistep first order method (see [4]) known as the heavy ball method. The name of this method derive from the fact that Eq. (1) can be interpreted as the equation of motion of an heavy ball with friction subject to the potential $V(w)$. Equation (1) is also closely related to the continuous approximation of other first order methods (see [5]). More directly in the case $m \rightarrow 0$ and $\eta$ fixed we get the continuous version of a plain gradient descent method with learning rate $1 / \eta$ :

$$
\dot{w}=-\frac{1}{\eta} \nabla V(w) .
$$

Notice the importance of the first order term in Eq. (1); without dissipation we wouldn't be able to recover the classical gradient descent method. Even worse, in general without the presence of the $\eta$ term there is no hope for the dynamic to reach a stationary point of $V$. Indeed, broadly speaking, since in that case the mechanical energy would be conserved lower values of $V$ correspond to higher values of the velocity so that the system do not have any chance to settle in a minimum of the potential.

More generally, as we already stated we believe that this "dynamical" approach to ML can be particularly fruitful when we want to consider online learning problems, that is to say problems where the temporal evolution of the parameters of the model at a certain stage of development depends explicitly on the data presented to the agent at the same time. This means that it is particularly important to handle the case in which the potential depends on time also trough a signal $u(t)$. Under this assumption Eq. (1) assumes the form

$$
m \ddot{w}(t)+\eta \dot{w}(t)+\nabla U(w(t), u(t))=0 .
$$

This equation, in the limit $m \rightarrow 0$ yields 


\begin{tabular}{ccl}
\hline \multicolumn{2}{l}{ Learning } & Mechanics \\
\hline$w$ & $\boldsymbol{q}$ & Weights and neuronal outputs are interpreted as generalized coordinates \\
$\dot{w}$ & $\dot{\boldsymbol{q}}$ & $\begin{array}{l}\text { Weight variations and neuronal variations are interpreted as generalized } \\
\text { velocities. }\end{array}$ \\
\hline
\end{tabular}

Table 1 Links between learning theory and classical mechanics.

$$
\dot{w}(t)=-\frac{1}{\eta} \nabla U(w(t), u(t)),
$$

that can be interpreted as the continuous counterpart of a stochastic gradient descent method, when $u(t)$ is interpreted as the realization of the random variable associated with the data at the step $t$. It is important to realize that whereas SGD is typically used in ML assuming that the values of $u(t)$ are drawn from a training set according to some probability distribution it is only when formulating the problem using a signal $u(t)$ which has a temporal regularity (coherence) that we can properly speak of online learning.

The paper is organized as follows: In Section 2 we will show how to reformulate least action principles in a more precise manner following what has been done in [6], Section 3 then shows how to extend some of the results of [6] (namely the existence of the minimum for approximating problems) also in the particularly interesting case where the potential explicitly depends on time. Eventually Section 4 closes the paper with some final considerations.

\section{Lagrangian Mechanics}

Following the approach proposed in [6], we will now discuss how it is possible to reformulate, in a more precise manner, the least action principle in classical mechanics. The following approach can be directly applied, in the case of dissipative dynamics, to learning processes simply through the identification of the generalized coordinates of mechanics with the parameters of the learning model (Table 1). In the remainder of the paper we will replace the variable $w$ which we used in the introduction to stress the connection with the typical parameters (weights) used in ML with the generic coordinates $\boldsymbol{q}$.

Usually (see [7] and [8]) Hamilton's principle is formulated as follows: Newton's laws of motion

$$
\frac{d}{d t}\left(m \dot{\boldsymbol{q}}_{i}(t)\right)+\nabla_{i} V(\boldsymbol{q}(t))=0,
$$

coincide with extremals of the functional

$$
\mathrm{S}(\boldsymbol{q}):=\int_{0}^{T} L d t, \quad \text { where } \quad L=\frac{1}{2} m|\dot{\boldsymbol{q}}|^{2}-V(\boldsymbol{q}),
$$


where $|\cdot|$ is the $n$-dimensional Euclidean norm. This statement is usually also called least action principle even though it is well known that the trajectory $\boldsymbol{q}(t)$ is not always a minimum for the action. Another unsatisfactory aspect of this principle is the way in which the initial conditions are handled; in newtonian mechanics Eq. (2) is typically coupled with Cauchy initial conditions

$$
\boldsymbol{q}(0)=\boldsymbol{q}^{0}, \quad \dot{\boldsymbol{q}}(0)=\boldsymbol{q}^{1},
$$

that uniquely determine the motion of the system. On the other hand Eq. (2) cannot be obtained from Hamilton's principle with conditions (4); usually the derivations make use of Dirichlet boundary conditions (see [7]).

It has been shown (in [6]) that Hamilton's principle can be replaced by a minimization problem together with a limiting procedure. In particular, let us consider the functionals

$$
\mathrm{W}_{\varepsilon}(\boldsymbol{q}):=\int_{0}^{T} e^{-t / \varepsilon}\left(\frac{\varepsilon^{2} m}{2}|\ddot{\boldsymbol{q}}(t)|^{2}+V(\boldsymbol{q}(t))\right) d t
$$

defined on the set dom $\mathrm{W}_{\varepsilon}:=\left\{\boldsymbol{q} \in H^{2}\left((0, T) ; \mathbb{R}^{n}\right) \mid \boldsymbol{q}(0)=\boldsymbol{q}^{0}, \dot{\boldsymbol{q}}(0)=\boldsymbol{q}^{1}\right\}$, where $V \in C^{1}\left(\mathbb{R}^{n}\right)$ and bounded from below and $m>0$.

The first property of this functional is that it admits a minimizer on its domain; actually adding little bit of regularity on $V$ and choosing $\varepsilon$ sufficiently small the minimizer turns out to be unique (for a precise statement of this result see Lemma 4.1 of [6]). Moreover the Euler-Lagrange equations for the minimizers of $\mathrm{W}_{\varepsilon}$ are (see Section 4 of [6])

$$
\begin{aligned}
& \varepsilon^{2} m \boldsymbol{q}^{(4)}(t)-2 \varepsilon m \boldsymbol{q}^{(3)}(t)+m \ddot{\boldsymbol{q}}(t)+\nabla V(\boldsymbol{q}(t))=0 \quad t \in(0, T), \\
& \boldsymbol{q}(0)=\boldsymbol{q}^{0}, \quad \dot{\boldsymbol{q}}(0)=\boldsymbol{q}^{1}, \\
& \ddot{\boldsymbol{q}}(T)=\boldsymbol{q}^{(3)}(T)=0 .
\end{aligned}
$$

Notice that from the stationarity condition of (5) we get two extra boundary conditions at time $t=T$ that seems to destroy causality of the solution; one of the strengths of this approach however is that, unlike Hamilton Principle, the boundary conditions (8) will disappear in the limit $\varepsilon \rightarrow 0$ leaving the solution dependent only on the initial state.

In the same limit $\left(\varepsilon \rightarrow 0\right.$ ), we have that if $\boldsymbol{q}_{\varepsilon}$ solves (6)-(8), then (Theorem 4.2 of [6]) $\boldsymbol{q}_{\varepsilon} \rightarrow \boldsymbol{q}$ weakly in $H^{1}\left((0, T) ; \mathbb{R}^{n}\right)$, where $\boldsymbol{q}$ solves (2) with (4). This last assertion makes clear that Hamilton principle can be reformulated in terms of (5) in the following way:

1. For each fixed $\varepsilon$ minimize $\mathrm{W}_{\varepsilon}$,

2. take the limit $\varepsilon \rightarrow 0$.

Like Hamilton's principle this procedure is a variational approach to classical mechanics, with respect to the principle of least cognitive action however, as anticipated, it involves a true minimization of the functional (5) and it automatically reaches causality. 
It is interesting to notice that if we omit step 2. in the procedure described above, stationarity conditions of (5) would imply a dynamic based on differential equations of order higher than two (which has been actually considered in physics [9] and [10]). However the presence of the right boundary conditions $(8)$ for each $\varepsilon>0$ would render the resulting laws non-causal.

To conclude this section we will discuss what can be considered yet another advantage of this approach by showing how naturally it can handle dissipative dynamics.

Dissipative dynamics. In the introduction we have briefly discussed how dissipation is a fundamental feature for the formulation of learning ad as a dynamical process; for this reason this point deserves a careful discussion.

First of all notice that it is not possible to modify $L$ in Eq. (3) by choosing an appropriate $V$ or by adding additional derivative terms in order to reproduce the following dissipative dynamics:

$$
m \ddot{\boldsymbol{q}}+\eta \dot{\boldsymbol{q}}+\nabla V(\boldsymbol{q})=0
$$

with $\eta>0$. Nevertheless it has been shown (see [11] and [3]) that it is possible to include this kind of dynamic by the following modification of the action:

$$
\mathrm{S}(\boldsymbol{q}) \rightarrow \overline{\mathrm{S}}(\boldsymbol{q}):=\int_{0}^{T} e^{\eta t / m}\left(\frac{1}{2} m|\dot{\boldsymbol{q}}|^{2}-V(\boldsymbol{q})\right) d t .
$$

This formulation changes the structure of the action functional making it more similar to the $W_{\varepsilon}$ functional. Still this variational approach suffers of the same problems that has been discussed previously in this section.

On the other hand in order to include dissipation in (5) it is sufficient to modify the $\mathrm{W}_{\varepsilon}$ functional in the following way:

$$
\mathrm{W}_{\varepsilon}(\boldsymbol{q}) \rightarrow \overline{\mathrm{W}}_{\varepsilon}(\boldsymbol{q}):=\int_{0}^{T} e^{-t / \varepsilon}\left(\frac{\varepsilon^{2} m}{2}|\ddot{\boldsymbol{q}}(t)|^{2}+\frac{\varepsilon \eta}{2}|\dot{\boldsymbol{q}}(t)|^{2}+V(\boldsymbol{q}(t))\right) d t .
$$

Then through the same minimization and limiting procedure described above we recover Eq. (9) together with the correct initial conditions (4).

The modification $\mathrm{W}_{\varepsilon}(\boldsymbol{q}) \rightarrow \overline{\mathrm{W}}_{\varepsilon}(\boldsymbol{q})$ feels less artificial than $\mathrm{S}(\boldsymbol{q}) \rightarrow \overline{\mathrm{S}}(\boldsymbol{q})$ and the term added to $W_{\varepsilon}$ seems a natural term to add. The reason why the dissipative behaviour is recovered so easily by the variational approach based on $W_{\varepsilon}$ is that this principle is not invariant by time reversal to begin with.

\section{Generalization to time-dependent potential}

The analysis presented in this section extends the result on the existence of a minimizer to a family of functionals that include (5) where, in particular, we allow an explicit dependence on time through the potential. 
The following theory is relevant at least for two distinct reason; first of all it is a first result that goes in the direction of extending the theory presented in [6]. In second place it is interesting in its own (i.e. also if it is not coupled with a limiting procedure) to ensure well-posedness of theories that relies on the minimization of a functional of the form that we will consider. Recently learning theories based on variational indexes considered in this section has been used in Vision; in particular the proposed theory has been directly applied to the problem of feature extraction from a video signal $u(t)$ in an unsupervised manner with the potential $U$ chosen to be the mutual information between the visual data and a set of symbols (see [2]).

Let $T \in(0, \infty), U \in C^{0}\left(\mathbb{R}^{n} \times \mathbb{R}^{m}\right)$ be bounded from below such that $U(\cdot, 0) \equiv 0$ and $\varpi \in L^{\infty}(0, T)$ with $0<C_{1} \leq \varpi(t) \leq C_{2}<+\infty$ for a.e. $t \in(0, T)$. Let $u:[0,+\infty) \rightarrow \mathbb{R}^{m}$ be an external input function that for the moment can be considered a continuous function of time. Consider the functional

$$
\Gamma(\boldsymbol{q})=\int_{0}^{T} \varpi(t)\left(\frac{\mu}{2}|\ddot{\boldsymbol{q}}(t)|^{2}+\frac{v}{2}|\dot{\boldsymbol{q}}(t)|^{2}+\gamma \dot{\boldsymbol{q}}(t) \cdot \ddot{\boldsymbol{q}}(t)+\frac{\kappa}{2}|\boldsymbol{q}(t)|^{2}+U(\boldsymbol{q}(t), u(t))\right) d t,
$$

where $\mu=\alpha+\gamma_{2}^{2}, v=\beta+\gamma_{1}^{2}, \gamma=\gamma_{1} \gamma_{2}, \kappa>0$ are real numbers so that (10) can always be rewritten as

$\Gamma(\boldsymbol{q})=\int_{0}^{T} \varpi(t)\left(\frac{\alpha}{2}|\ddot{\boldsymbol{q}}(t)|^{2}+\frac{\beta}{2}|\dot{\boldsymbol{q}}(t)|^{2}+\frac{1}{2}\left|\gamma_{1} \dot{\boldsymbol{q}}(t)+\gamma_{2} \ddot{\boldsymbol{q}}(t)\right|^{2}+\frac{\kappa}{2}|\boldsymbol{q}(t)|^{2}+U(\boldsymbol{q}(t), u(t))\right) d t$,

with $\alpha, \beta$, real and positive and $\boldsymbol{q} \in \operatorname{dom}(\Gamma):=\left\{\boldsymbol{q} \in H^{2}\left((0, T) ; \mathbb{R}^{n}\right) \mid \boldsymbol{q}(0)=\right.$ $\left.\boldsymbol{q}^{0}, \quad \dot{\boldsymbol{q}}(0)=\boldsymbol{q}^{1}\right\}$, where $\boldsymbol{q}_{0}, \boldsymbol{q}_{1} \in \mathbb{R}^{n}$ are given.

Suppose furthermore that we equip $\operatorname{dom}(\Gamma)$ with the following notion of convergence:

$$
\begin{array}{ll}
\boldsymbol{q}_{k} \rightarrow \boldsymbol{q} & \text { strongly in } H^{1}\left((0, T) ; \mathbb{R}^{n}\right) ; \\
\ddot{\boldsymbol{q}}_{k} \rightarrow \ddot{\boldsymbol{q}} & \text { weakly in } L^{2}\left((0, T) ; \mathbb{R}^{n}\right) .
\end{array}
$$

Then the following remark holds:

Remark 1 The set $\operatorname{dom}(\Gamma)$ is closed under the convergence in (11), i.e., if $\boldsymbol{q}_{k} \in$ $\operatorname{dom}(\Gamma), \boldsymbol{q}_{k} \rightarrow \boldsymbol{q}$ in $\operatorname{dom}(\Gamma)$, then $\boldsymbol{q} \in \operatorname{dom}(\Gamma)$.

Indeed, since $H^{1}(0, T)$ compactly embeds in $C([0, T])$ (see [12] pag. 213 Eq. (6)) and a weakly convergence sequence is strongly bounded ([12] Prop. 3.5 (iii)), $\left\langle\boldsymbol{q}_{k}\right\rangle$ has a (not relabelled) subsequence such that $\boldsymbol{q}_{k} \rightarrow \boldsymbol{q}$ and $\dot{\boldsymbol{q}}_{k} \rightarrow \dot{\boldsymbol{q}}$ uniformly in $[0, T]$, therefore $\boldsymbol{q}(0)=\boldsymbol{q}^{0}$ and $\dot{\boldsymbol{q}}(0)=\boldsymbol{q}^{1}$.

We are now in the position to state the main result on the existence of a minimum of the functional in (10).

Theorem 1 The problem $\min \{\Gamma(\boldsymbol{q}) \mid \boldsymbol{q} \in \operatorname{dom}(\Gamma)\}$, has a solution.

Proof We simply apply the direct method in the calculus of variations, namely we have to show that $\Gamma$ is lower semicontinuous and coercive with respect to the convergence in (11) and then we conclude in view of Remark 1 
Lower semicontinuity. The maps $\boldsymbol{q} \in \operatorname{dom}(\Gamma) \mapsto \int \varpi(t)|\boldsymbol{q}(t)|^{2} d t$ and $\boldsymbol{q} \in$ $\operatorname{dom}(\Gamma) \mapsto \int \varpi(t)|\dot{\boldsymbol{q}}(t)|^{2} d t$ are continuous, while $\boldsymbol{q} \in \operatorname{dom}(\Gamma) \mapsto \int \varpi(t)|\ddot{\boldsymbol{q}}(t)|^{2} d t$ is lower semicontinuous (see [12] Prop. 3.5 (iii)); moreover $\boldsymbol{q} \in \operatorname{dom}(\Gamma) \mapsto$ $\int \varpi(t) \dot{\boldsymbol{q}}(t) \cdot \ddot{\boldsymbol{q}}(t) d t$ is continuous because of the strong-weak convergence of the scalar product in a Hilbert space (see [12] Prop. 3.5 (iv)). Finally the map $\boldsymbol{q} \in \operatorname{dom}(\Gamma) \mapsto \int \varpi(t) U(\boldsymbol{q}(t), u(t))$ is lower semicontinuous because of our assumptions on $U$ and as a direct consequence of Fatou's Lemma.

Coercivity. Since $U$ is bounded from below and $T<\infty$ and in view of our assumptions on $w, \alpha, \beta, \kappa$ it immediately follows that if $\sup _{k \in \mathbb{N}} \Gamma\left(\boldsymbol{q}_{k}\right)<+\infty$, then there exists a constant $C>0$ such that $\left\|\boldsymbol{q}_{k}\right\|_{H^{2}} \leq C$ for every $k \in \mathbb{N}$. Then from Theorem 3.16 in [12] it follows that $\left\langle\boldsymbol{q}_{k}\right\rangle$ has a subsequence weakly converging in $H^{2}(0, T)$. Moreover since $H^{2}(0, T)$ compactly embeds in $H^{1}(0, T)$ then there is a subsequence that converges strongly in $H^{1}(0, T)$. This means that indeed the sublevels of $\Gamma$ are compact with respect to the convergence in Eq. (11).

\section{Conclusions}

In this paper we presented an extension of the minimality result discovered in [6] that entails the well-posedness of a class of learning problems based on a Least Action Principle defined over the class of functionals (10). We prove that the existence of the minimum of $\Gamma$ (Theorem 1 ) holds for a general weight function $\varpi$. Moreover, we argue that since learning requires dissipation, the correspondent dynamics can be reproduced from (10) by choosing $\varpi$ as an exponential function of time, as discussed in Section 2. This paper provides motivations to use the variational framework initially proposed in [3], since it shows that, unlike the action of mechanics, the opportune selections of the cognitive action leads to well-posed learning problems where a global minimum can be discovered.

Acknowledgements We thank Giovanni Bellettini for having brought to our attention the extended formulation of Newtonian mechanics and for insightful discussions.

\section{References}

1. Weinberg, S.: The quantum theory of fields. Vol. 1: Foundations. Cambridge University Press (1995).

2. Betti, A., Gori, M., Melacci, S.: Cognitive Action Laws: The Case of Visual Features. arXiv:cs.CV/1808.09162v1, accepted for publication in the IEEE Trans. on Neural Networks and Learning Systems.

3. Betti, A. and Gori, M.: The principle of least cognitive action. Theoretical Computer Science, 633, 83-99 (2016).

4. Polyak, B.T.: Some methods of speeding up the convergence of iteration methods. USSR Computational Mathematics and Mathematical Physics, 4, 1-17 (1964). 
5. Su, W., Boyd, S., Candes, E.: A differential equation for modeling Nesterov's accelerated gradient method: Theory and insights. Advances in Neural Information Processing Systems, 2510-2518 (2014).

6. Liero, M. and Stefanelli U.: A new Minimum Principle for Lagrangian Mechanics. Journal of Nonlinear Science 23, 179-204 (2013)

7. Vladimir, I.: Arnold. Mathematical methods of classical mechanics. Graduate Texts in Mathematics, 60 (1989).

8. Goldstein, H., Poole, C. and Safko, J.: Classical mechanics. Addison Wesley (2002).

9. Suykens, J. A. K: Extending Newton's law from nonlocal-in-time kinetic energy. Physics Letters A, 373(14), 1201-1211 (2009).

10. El-Nabulsi, R. A.: On maximal acceleration and quantum acceleratum operator in quantum mechanics. Quantum Studies: Mathematics and Foundations, 5(4), 543-550 (2018).

11. Herrera, L., Nunez, L., Patino, A. and Rago, H.: A variational principle and the classical and quantum mechanics of the damped harmonic oscillator. American Journal of Physics, 54(3), 273-277 (1986).

12. Brezis, H.: Functional Analysis, Sobolev Spaces and Partial Differential Equation. Springer Science \& Business Media (2010) 